Draft Version November 20, 2018

Preprint typeset using LATEX style emulateapj v. 5/2/11

\title{
HOW EMPTY ARE DISK GAPS OPENED BY GIANT PLANETS?
}

\author{
JEFFREY Fung (馮澤之) ${ }^{1}$, Ji-Ming SHI (史集明) ${ }^{2}$, AND EUGENE ChIANG (蔣詒曾) ${ }^{2,3}$ \\ Draft version November 20, 2018
}

\begin{abstract}
Gap clearing by giant planets has been proposed to explain the optically thin cavities observed in many protoplanetary disks. How much material remains in the gap determines not only how detectable young planets are in their birth environments, but also how strong co-rotation torques are, which impacts how planets can survive fast orbital migration. We determine numerically how the average surface density inside the gap, $\Sigma_{\text {gap }}$, depends on planet-to-star mass ratio $q$, Shakura-Sunyaev viscosity parameter $\alpha$, and disk height-to-radius aspect ratio $h / r$. Our results are derived from our new GPU-accelerated Lagrangian hydrodynamical code PEnGUIn, and are verified by independent simulations with ZEUS90. For Jupiter-like planets, we find $\Sigma_{\text {gap }} \propto$ $q^{-2.2} \alpha^{1.4}(h / r)^{6.6}$, and for near brown dwarf masses, $\Sigma_{\text {gap }} \propto q^{-1} \alpha^{1.3}(h / r)^{6.1}$. Surface density contrasts inside and outside gaps can be as large as $10^{4}$, even when the planet does not accrete. We derive a simple analytic scaling, $\Sigma_{\text {gap }} \propto q^{-2} \alpha^{1}(h / r)^{5}$, that compares reasonably well to empirical results, especially at low Neptune-like masses, and use discrepancies to highlight areas for progress.

Subject headings: accretion, accretion disks — methods: numerical — planets and satellites: formation protoplanetary disks — planet-disk interactions
\end{abstract}

\section{INTRODUCTION}

Observational studies of giant planet formation will begin in earnest once we detect planets still embedded in their natal gas disks. Directly imaging young gas giants is made easier by their ability to clear material away from their orbits. Planetary (Lindblad) torques open gaps while viscous torques fill them back in (Goldreich and Tremaine 1980): a balance between these torques sets the equilibrium surface density near the planet. There are hints of gap clearing by planets in so-called "transitional" disks having optically thin cavities (e.g., Kraus and Ireland 2012; Debes et al. 2013; Ouanz et al. 2013). Transition disk holes are surprisingly large; one gap opened by a single planet would be too narrow to explain the observed cavity sizes that range up to $\sim 100 \mathrm{AU}$, and so a given system might have to contain multiple super-Jovian planets to clear a wide enough swath (e.g., Zhu et al. 2011; Dodson-Robinson and Salyk 2011). Even so, the holes are so optically thin that planets alone seem incapable of torquing material strongly enough to compete with viscous diffusion - at least for typically assumed parameters - and appeals are made to opacity reductions through grain growth or dust filtration at the outer gap edge (Zhu et al. 2012; Dong et al. 2012). Part of the motivation of our study is to expand the parameter space explored and see how empty a gap can be cleared.

Determining gap surface densities and corresponding optical depths is relevant not only for observations but also for theory: material that co-rotates with the planet (executing quasi-horseshoe orbits) can backreact gravitationally on the planet and influence its dynamical evolution. The co-rotation torque takes its place among the litany of resonant planet-disk interactions that can alter orbital eccentricities and semima-

Electronic address: fung@ astro.utoronto.ca

${ }^{1}$ Department of Astronomy and Astrophysics, University of Toronto, 50 St. George Street, Toronto, Ontario, Canada M5S 3H4

2 Department of Astronomy, UC Berkeley, Hearst Field Annex B-20, Berkeley, CA 94720-3411

${ }^{3}$ Department of Earth and Planetary Science, UC Berkeley, $307 \mathrm{Mc}-$ Cone Hall, Berkeley, CA 94720-4767 jor axes (for a review, see Kley and Nelson 2012). The delicate balance of forces within the gap, including the thermodynamic behavior of matter there, may determine how low-mass and giant planets survive the threats of Types I and II orbital migration (Ward 1997; see also section 2.2 of Kley and Nelson 2012 and references therein).

Despite its importance, $\Sigma_{\text {gap }}$ - the surface density averaged over the bottom of the gap - remains poorly understood. Notwithstanding the huge number of simulations of planet-disk interactions published in the past two decades ${ }_{4}^{4}$ a systematic parameter study has yet to be performed that determines $\Sigma_{\text {gap }}$ as a function of planet-to-star mass ratio $q \equiv M_{p} / M_{*} ;$ Shakura-Sunyaev viscosity parameter $\alpha$; and disk height-to-radius aspect ratio (equivalently, disk temperature) $h / r$. Crida et al. (2006) examined how these parameters influence gap shape and width, but not gap depth - i.e., they studied the onset of the gap, but not the bottom of the gap. The numerics can be challenging. Measuring $\Sigma_{\text {gap }}$ accurately requires global simulations that (i) resolve the disk well in at least azimuth $\phi$ and radius $r$; (ii) resolve large density contrasts; (iii) converge with time to a steady state; (iv) model how the planet accretes from the ambient flow; and (v) possess well-separated radial boundaries that maintain a steady mass accretion rate across the entire domain, i.e., the simulation presumably should enforce $\dot{M}(r)=$ constant $\neq 0$, as befits real accretion disks. Feature (v) is captured by only a minority of studies, and feature (iv) can only be mocked up in a parameterized way (e.g., Lubow and D'Angelo 2006; Zhu et al. 2011).

This paper aims to provide an empirical relation for $\Sigma_{\text {gap }}(q, \alpha, h / r)$ for a single non-accreting giant planet on a fixed circular orbit embedded in a 2D, locally isothermal, steadily accreting disk. We restrict our study to disk gas only, and ignore how dust and gas flows might differ. We utilize

\footnotetext{
${ }^{4}$ Much of the literature is marked by a peculiar insistence on plotting surface density $\Sigma$ on a linear scale. The practice is unhelpful since surface density contrasts in and out of gaps can span orders of magnitude - indeed they must if they are to reproduce the enormous optical depth contrasts inferred from observations of transition disks (e.g., Dong et al. 2012).
} 
two independent codes: ZEUS3D (Stone and Norman 1992), and PEnGUIn, a new Lagrangian PPM (piecewise parabolic method)-based code that we have implemented on multiple GPUs (graphics processing units). To the extent possible, results from one code will be validated against the other.

\subsection{An Analytic Scaling Relation}

Although our study is primarily numerical, we derive here an approximate analytic relation for $\Sigma_{\text {gap }}$ that we will use to put our numerical results in context. We admit at the outset that our derivation can hardly be called such, as our reasoning will ignore many details and make assumptions not carefully justified. But as the rest of our paper will show, the simple relation we now present will yield results surprisingly close to those of detailed numerical simulations.

First examine the outer disk, exterior to the planet's orbit. The outer Lindblad torque exerted by the planet transmits angular momentum outward at a rate:

$$
T_{\mathrm{L}} \sim q^{2}\left(\frac{r}{h}\right)^{3} \Sigma_{\text {gap }} \Omega^{2} r^{4}
$$

where $\Omega$ is the disk angular frequency (Goldreich and Tremaine 1980). This is the total torque from linear perturbations to one side of the planet, integrated over all resonances up to the torque cut-off at azimuthal wavenumber $m \sim r / h$ (see, e.g., equation 2 of Crida et al. 2006, and references therein). Note that we have used $\Sigma_{\text {gap }}$, the surface density averaged over the bottom of the gap, in our evaluation of the integrated Lindblad torque. Our justification for this choice is that the integrated torque is dominated by resonances at the torque cut-off, i.e., at distances $\sim h$ from the planet (see figures 2 and 3 of Goldreich and Tremaine 1980), and bottoms of gaps opened by giant planets in gas disks typically extend this far (see, e.g., the simulated gaps of Crida et al. 2006, or our Figure 2). One caveat is that the linear theory from which equation (1) originates is formally valid only for low-mass planets for which $q \lesssim(h / r)^{3}$; the highest-mass planets we simulate will violate this condition, and indeed for such super-Jovian objects our numerical simulations will reveal deviations from the simple-minded scaling law we derive in this section.

In steady state, the outward transmission of angular momentum by the (outer) Lindblad torque must be balanced by the angular momentum transmitted inward by the viscous torque 5

$$
T_{\mathrm{v}} \sim \Sigma_{0} v \Omega r^{2}
$$

where $v=\alpha(h / r)^{2} \Omega r^{2}$ is the kinematic viscosity (e.g., Frank et al. 2002). Here we have used $\Sigma_{0}$, the surface density at the gap periphery - or more conveniently, the surface density at the planet's location if the planet were massless to evaluate $T_{\mathrm{v}}$. The viscous torque depends on the gradient of $\Sigma$, and this gradient is larger outside the flat-bottomed gap than inside it (see, e.g., the gap profile shown in Figure 2, by "gap periphery" we mean a location like $r \approx 1.4$, where the gradient might reasonably be approximated as $\Sigma_{0} / r$, which is what equation 2 essentially assumes). Conscripting $\Sigma_{0}$ in this way is a gross simplification, but alternatives would require that we actually compute the precise shape of the gap, which

\footnotetext{
5 There is also a so-called "pressure" torque of comparable magnitude to the Lindblad and viscous torques (Crida et al. 2006), but we neglect this third torque for our order-of-magnitude derivation.
}

is what we are trying to avoid with our order-of-magnitude derivation.

Usually one thinks of viscous torques as transmitting angular momentum outward, but in the gap edge of the outer disk, the direction of viscous transport is inward because the surface density there has a sharp and positive gradient $(d \Sigma / d r>0)$.

Setting $T_{\mathrm{L}}=T_{\mathrm{v}}$ yields

$$
\frac{\Sigma_{\text {gap }}}{\Sigma_{0}} \sim \frac{\alpha(h / r)^{5}}{q^{2}} .
$$

Exactly the same scaling relation applies to the inner disk, interior to the planet's orbit. The signs in the inner disk are reversed from those in the outer disk: the inner Lindblad torque transmits angular momentum inward, while the local viscous torque transmits angular momentum outward.

As we were completing our numerical tests of equation (3) and preparing our manuscript for publication, we became aware of the study by Duffell and MacFadyen (2013) who found the same scaling relation on purely empirical grounds (although these authors did not explicitly vary the Mach number $r / h)$. Duffell and MacFadyen (2013) concentrated on the low-mass $q \lesssim 10^{-4}$ regime. Our study complements theirs by studying the high-mass $q \gtrsim 10^{-4}$ regime; we will see to what extent equation (3) also holds true for giant planets. See also our $\$ 4.3$ where we discuss to what extent the short derivation given in this subsection captures the whole story.

\subsection{Plan of This Paper}

Section 2 contains our numerical methods and simulation parameters. Section 3 presents our results for $\Sigma_{\text {gap }}(q, \alpha, h / r)$. Section 4 concludes and charts directions for future work.

\section{NUMERICAL METHODS}

We numerically simulate a planet on a fixed circular orbit embedded in a co-planar, viscously accreting disk. Our two independent codes, PEnGUIn and ZEUS3D, solve the usual mass and momentum equations in two dimensions; we give here the equations in the inertial, barycentric frame (although neither code actually works in this frame; see $\$ 2.1$ and $\$ 2.2$ for the technical details):

$$
\begin{gathered}
\frac{D \Sigma}{D t}+\Sigma(\nabla \cdot \mathbf{v})=0, \\
\frac{D \mathbf{v}}{D t}=-\frac{1}{\Sigma} \nabla P+\frac{1}{\Sigma} \nabla \cdot \mathbf{T}-\nabla \Phi,
\end{gathered}
$$

where $D / D t$ is the Lagrangian derivative, $\Sigma$ is the surface density, $P$ is the vertically integrated pressure, $\mathbf{v}$ is the velocity, $\mathbf{T}$ is the Newtonian viscous stress tensor, and $\Phi$ is the gravitational potential of the central star and planet (but not the disk). In polar coordinates, $\mathbf{v}=\left(v_{r}, \Omega r\right)$; in component form, equation (5) reads: 


$$
\begin{aligned}
\frac{D v_{r}}{D t}= & -\frac{1}{\Sigma} \frac{\partial P}{\partial r}+\frac{2}{\Sigma r} \frac{\partial}{\partial r}\left(v \Sigma r \frac{\partial v_{r}}{\partial r}\right) \\
& +\frac{1}{\Sigma r} \frac{\partial}{\partial \phi}\left[v \Sigma\left(r \frac{\partial \Omega}{\partial r}+\frac{1}{r} \frac{\partial v_{r}}{\partial \phi}\right)\right]-\frac{\partial \Phi}{\partial r}, \\
\frac{D(r \Omega)}{D t}= & -\frac{1}{\Sigma r} \frac{\partial P}{\partial \phi}+\frac{2}{\Sigma r} \frac{\partial}{\partial \phi}\left(v \Sigma \frac{\partial \Omega}{\partial \phi}\right) \\
& +\frac{1}{\Sigma r^{2}} \frac{\partial}{\partial r}\left[v \Sigma r^{2}\left(r \frac{\partial \Omega}{\partial r}+\frac{1}{r} \frac{\partial v_{r}}{\partial \phi}\right)\right]-\frac{1}{r} \frac{\partial \Phi}{\partial \phi} .
\end{aligned}
$$

Here $v=\alpha c_{\mathrm{s}} h$ is the kinematic viscosity following Shakura and Sunyaev (1973), with $c_{\mathrm{s}}$ equal to the sound speed. We complete the equation set with a locally isothermal equation of state $P=\Sigma c_{\mathrm{s}}^{2}$, with $c_{\mathrm{s}} \propto r^{-1 / 2}$ so that the disk aspect ratio $h / r=$ constant.

In the center-of-mass frame,

$$
\begin{gathered}
\Phi=-\frac{G M_{*}}{\sqrt{r^{2}+r_{1}^{2}+2 r r_{1} \cos \left(\phi-\phi_{\mathrm{p}}\right)}} \\
-\frac{G M_{\mathrm{p}}}{\sqrt{r^{2}+r_{2}^{2}-2 r r_{2} \cos \left(\phi-\phi_{\mathrm{p}}\right)+r_{\mathrm{s}}^{2}}},
\end{gathered}
$$

where $M_{*}$ and $M_{\mathrm{p}}=q M_{*}$ are the masses of the star and the planet, respectively; $r_{1}=q r_{\mathrm{p}} /(1+q)$ and $r_{2}=r_{\mathrm{p}} /(1+q)$ are their radial positions, with $r_{\mathrm{p}}$ the total (fixed) separation; $\phi_{\mathrm{p}}-\pi$ and $\phi_{\mathrm{p}}$ are their angular positions; and $r_{\mathrm{s}}$ is the smoothing (a.k.a. softening) length of the planet's potential. We set $G\left(M_{*}+M_{\mathrm{p}}\right)=1$ and $r_{\mathrm{p}}=1$ so that the planet's orbital frequency $\Omega_{\mathrm{p}}=1$ and period $P_{\mathrm{p}}=2 \pi$.

\subsection{PEnGUIn: Code Description}

PEnGUIn (Piecewise Parabolic Hydro-code Enhanced with Graphics Processing Unit Implementation) is a Lagrangian, dimensionally-split, shock-capturing hydrodynamics code. We defer a detailed description to a future paper, and here just mention a few salient points. The code is written in CUDA-c and runs on multiple GPUs (graphics processing units) for accelerated performance. It uses the piecewise parabolic method (PPM; Colella and Woodward 1984), and its main solver is modelled after VH-1 (Blondin and Lufkin 1993) with two main differences: (i) PEnGUIn explicitly conserves angular momentum by replacing, as one of the quantities a fluid element carries in the Lagrangian frame, the angular speed with the specific angular momentum, and (ii) PEnGUIn uses a non-iterative Riemann solver for isothermal flows; see Balsara (1994), and note that their Lagrangian remap formulation applies not only to strictly isothermal flows, but also to locally isothermal flows under the two-shock approximation by allowing for different sound speeds for leftand right-moving waves. PEnGUIn also contains a module that computes the divergence of the stress tensor through piecewise parabolic interpolations.

Technically, PEnGUIn's reference frame is a barycentric frame that rotates at $\Omega_{\mathrm{p}}$; thus the planet's position is fixed in time, but the Coriolis force is not computed as an explicit source term. Rather, it is absorbed into the conservative form of the angular momentum equation (Klev 1998).

GPU-acceleration is optimal for programs that are massively parallel; PEnGUIn achieves parallelization through memory management and domain splitting. The hardware used for this paper are three GTX-Titan graphics cards all connected to a single node. Running in double precision on all three cards simultaneously, PEnGUIn takes 12 seconds to run per planetary orbit for $(q, \alpha, h / r)=\left(10^{-3}, 10^{-2}, 0.05\right)$.

\subsection{ZEUS90: Code Description}

For comparison with PEnGUIn, we also carried out simulations with ZEUS90: a modern version of ZEUS3D (Stone and Norman 1992; Hawley and Stone 1995) written in FORTRAN 90. It is a three-dimensional, operator-split, timeexplicit, Eulerian finite-differencing magnetohydrodynamics code, widely used to simulate a variety of systems, including magnetorotationally-unstable circumbinary disks (Shi et al. 2012) and warped disks (Sorathia et al. 2013). For our application, we suppress the vertical dimension and magnetic fields; implement the Navier-Stokes viscosity module; and add a planetary potential having a specified time dependence. The von Neumann-Richtmyer artificial viscosity, commonly used to capture shock waves, is switched off in the presence of an explicit viscosity. The reference frame for ZEUS90 is a non-rotating frame centered on the star, and so ordinarily there is an extra term in $\Phi$ due to the indirect potential: $G M_{\mathrm{p}} r \cos \left(\phi-\phi_{\mathrm{p}}\right) / r_{\mathrm{p}}^{2}$. However, we find in practice that the indirect term results in a "wobbling" of the disk that is difficult to reconcile with our fixed boundary conditions on fixed circles (see equations 9, 11 below and surrounding discussion). The wobbling generates spurious time variability that increases with increasing $q$; therefore we drop the indirect potential in all ZEUS90 simulations with $q \geq 1 \times 10^{-3}$ (apparently Lubow et al. 1999 also dropped the indirect potential in their simulations with ZEUS; see also Zhu et al. 2011 who found that their planet-disk simulations were not sensitive to the indirect term). Running in double precision on 128 cores in parallel, ZEUS90 takes 26 seconds to run per planetary orbit for $(q, \alpha, h / r)=\left(10^{-3}, 10^{-2}, 0.05\right)$.

\subsection{Numerical Setup}

For our parameter study we vary:

- $q$ from $10^{-4}$ to $10^{-2}$,

- $\alpha$ from $10^{-3}$ to $10^{-1}$, and

- $h / r$ from 0.04 to 0.1 .

Other properties of our simulations are as follows.

\subsubsection{Initial and boundary conditions}

Our simulation domain spans 0 to $2 \pi$ in azimuth, and from $r_{\text {in }}=0.4$ to $r_{\text {out }}=2.5$ in radius (in units where the planet-star separation $r_{\mathrm{p}}=1$ ). Initial conditions correspond to a steadystate accretion disk having constant $\alpha$, constant $h / r$, and a rotation curve modified by the radial pressure gradient:

$$
\begin{aligned}
& \Sigma=\Sigma_{0}\left(r / r_{\mathrm{p}}\right)^{-1 / 2}, \\
& v_{r}=-\frac{3}{2} \alpha\left(\frac{h}{r}\right)^{2} \sqrt{\frac{G\left(M_{*}+M_{\mathrm{p}}\right)}{r}}, \\
& \Omega=\sqrt{1-\frac{1}{2}\left(\frac{h}{r}\right)^{2}} \sqrt{\frac{G\left(M_{*}+M_{\mathrm{p}}\right)}{r^{3}}},
\end{aligned}
$$

with $\Sigma_{0}=1$ (we could have chosen any value for $\Sigma_{0}$ because we do not calculate the gravity of the disk- the disk exerts 


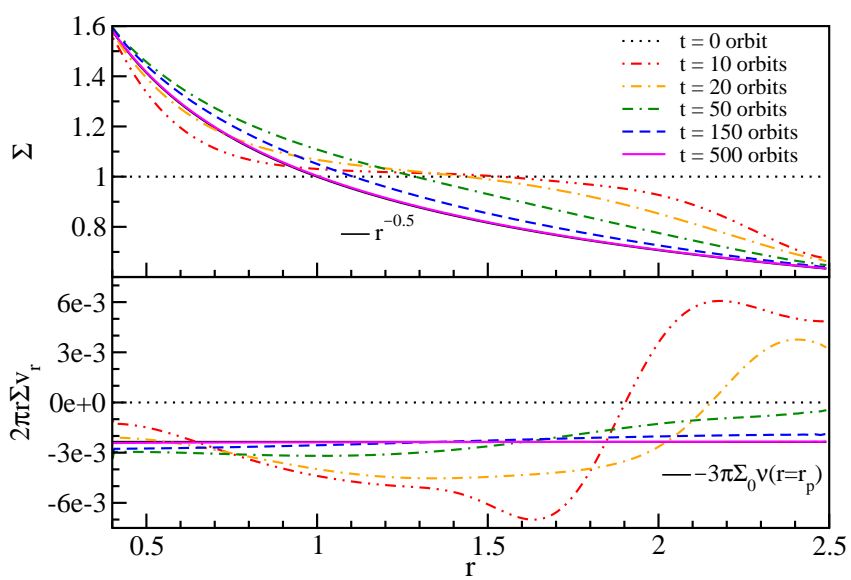

FIG. 1. - Viscous relaxation to steady-state accretion in a disk with $(q, \alpha, h / r)=(0,0.1,0.05)$. At $t=0$, we set $\Sigma=1$ and $v_{r}=0$ except at the boundaries, where conditions are given by equations 9-11. Black solid lines denote the steady-state density profile and accretion rate to which PEnGUIn correctly relaxes over a viscous timescale.

no gravitational backreaction on the planet nor does it selfgravitate). At both inner and outer radial boundaries, we fix $\Sigma, v_{r}$ and $\Omega$ to their values determined by the above equations. These fixed boundary conditions ensure a steady inflow of mass across the simulation domain — as is appropriate for real accretion disks 6 Figure 1 illustrates how our boundary conditions enable a planet-less disk to relax over a viscous diffusion timescale to the equilibrium profile described by equations (9)-11).

Ideally the radial boundaries should be placed far enough away that waves launched from the planet damp before they reach the edges of the domain. Goodman and Rafikov (2001) calculated that nonlinear steepening of waves causes them to dissipate over lengthscales of $\sim 3 h$ (for $q=10^{-3}$ and $h / r=0.05$; the damping length scales as $q^{-0.4}$ and $\left.(h / r)^{2.2}\right)$. Our outer radial boundary of $r_{\text {out }}=2.5(\sim 15-40 h$ away from the planet, depending on our choice for $h / r$ ) is distant enough that outward-propagating waves largely dissipate within the domain. For our inner radial boundary of $r_{\text {in }}=0.4(\sim 6-$ $15 h$ away from the planet), the situation is more marginal; depending on the simulation, waves are still present at our disk inner edge. However, the main focus of our paper is the surface density deep within the gap, and we have verified that this quantity changes by no more than $\sim 10 \%$ as we shrink $r_{\text {in }}$ from 0.4 to 0.2 . Thus we opt for the larger boundary to keep code timesteps longer. For simplicity we eschew wavekilling zones (cf. de Val-Borro et al. 2006). In practice, the Godunov-type scheme used by PEnGUIn is effective at absorbing waves at fixed boundaries, even more so than using wave-killing zones (Zhaohuan Zhu 2013, personal communication).

To avoid strong shocks at the beginning of the simulation, the planet mass is ramped from zero to its assigned value over an initial "warm-up" phase. In PEnGUIn, $M_{\mathrm{p}}$ increases according to $M_{\mathrm{p}}(t) / M_{*}=q \sin ^{2}\left[\left(\Omega_{\mathrm{p}} t / 20\right)\left(10^{-3} / q\right)\right]$. For $q=10^{-3}$, this takes 5 orbits. In ZEUS90, the planet mass grows linearly from zero to its desired value in 1 orbit. Both warm-up schemes proved stable.

\subsubsection{Grid resolution}

6 By contrast, many popular codes for planet-disk simulations (e.g., FARG0) default to a zero-inflow solution; for a compilation of codes from the community, see, e.g., de Val-Borro et al. (2006).

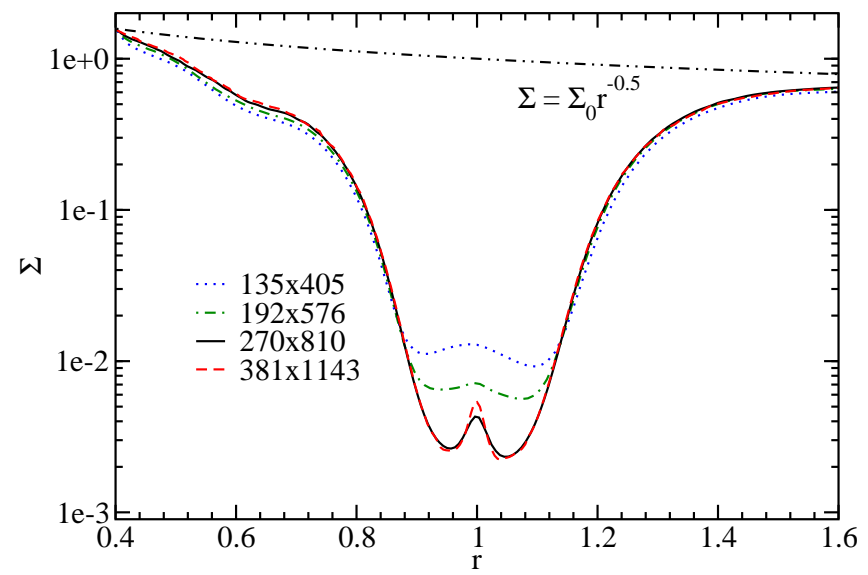

FIG. 2.- Convergence of gap profile with grid resolution for $(q, \alpha, h / r)=$ $\left(10^{-3}, 10^{-3}, 0.05\right)$ using PEnGUIn. The dot-dot-dashed curve represents the initial density profile, equal to the density profile in the absence of the planet (equation 9). The surface density $\Sigma$ plotted here is azimuthally averaged. For PEnGUIn science runs, we adopt $270(r) \times 810(\phi)$ for $h / r=0.05$, and adjust the cell size to scale with $h / r$ (see $\$ 2.3 .2$ ).

Our grid spacings are logarithmic in radius and uniform in azimuth. For $h / r=0.05$, the resolution is $270(r) \times 810(\phi)$ for PEnGUIn and $256 \times 864$ for ZEUS90 (the latter choice yields square grid cells). Figure 2 attests that gap surface densities have largely converged at our standard resolution. We scale our grid cell size with $h / r$, i.e., with sound speed $c_{\mathrm{s}}$, so that sound waves of a given frequency are equally well resolved between simulations. Cold disks with small $h / r$ are especially costly, which is why we do not vary $h / r$ below 0.04 . Code timesteps scale with grid cell sizes according to the CourantFriedrichs-Lewy condition, with the Courant number chosen to be 0.5 for PEnGUIn and 0.4 for ZEUS90.

\subsubsection{Smoothing length $r_{s}$}

For both PEnGUIn and ZEUS90, the planetary potential's softening length is fixed at $r_{s}=0.028 r_{\mathrm{p}}$ or about 4 local grid cell lengths. Equivalently, $r_{s}=0.56 h$ for $h / r=0.05$, and $r_{s}=0.25$ Hill radii $R_{\mathrm{H}}$ for $q=10^{-3}$. Any choice for $r_{s} \sim h$ or $r_{s} \sim R_{\mathrm{H}}$ seems reasonable insofar as our 2D treatment of the gas dynamics must break down at distances from the planet less than the vertical thickness of the disk, and because the planet's mass may, in reality, be distributed over a distended envelope or circumplanetary disk. Tests with PEnGUIn at $(q, \alpha, h / r)=\left(10^{-3}, 0.1,0.05\right)$ revealed that $r_{s} \lesssim 0.4 h$ caused the surface density to converge substantially more slowly with time. Specifically, for the aforementioned parameters and $r_{s}$ too small, the gap deepened rapidly, overshot its equilibrium value, and took thousands of orbits to approach a steady state. By contrast, for $r_{s}=0.56 h$, the surface density equilibrated in a mere $\sim 30$ orbits at our standard resolution, with higher grid resolutions yielding similar results.

According to Müller et al. (2012), our choice of smoothing length yields a $2 \mathrm{D}$ gravitational force that matches the vertically averaged, 3D force to within $10 \%$ at a distance $\gtrsim 2 h$ away from the planet.

\subsection{4. $\Sigma_{\text {gap }}$ and convergence with time}

Our metric for gap depth is the space- and time-averaged surface density $\Sigma_{\text {gap }}$ in the planet's co-rotation region, normalized to $\Sigma_{0}=1$ (the surface density at $r=r_{\mathrm{p}}=1$ in the absence of the planet). As judged from snapshots like those shown in Figures 3 and 4 the annulus spanning $r=r_{\mathrm{p}}-\Delta$ to $r_{\mathrm{p}}+\Delta$ with $\Delta \equiv 2 \max \left(R_{\mathrm{H}}, h\right)$, excised from $\phi=\phi_{\mathrm{p}}-\Delta / r_{\mathrm{p}}$ 
to $\phi_{\mathrm{p}}+\Delta / r_{\mathrm{p}}$, is visibly depleted and reasonably uniform. For most simulations, this is the area over which we average $\Sigma$ to calculate $\Sigma_{\text {gap }}$.

In a few cases the outer edge of the gap is visibly eccentric (see $\$ 3.2$ for more discussion), and the circular annulus we have defined above becomes contaminated with non-gap material and is no longer suitable for measuring $\Sigma_{\text {gap }}$. In these cases, we keep the circular inner gap edge and the azimuthal excision as defined above, but approximate the outer gap edge with an ellipse having semimajor axis $r_{\mathrm{p}}+\Delta$, and an eccentricity and apsidal orientation estimated by eye from snapshots (for a sampling, jump to Figures 10 and 11).

Each simulation runs until $\Sigma_{\text {gap }}$ appears to have converged in time; see Figure 5 for an example. The time required to reach convergence scales approximately as the viscous timescale $r_{\mathrm{p}}^{2} / v$, shortening somewhat with larger $q$. Each value of $\Sigma_{\text {gap }}$ that we report in Table 1 is averaged over a time interval that starts at time $t_{\text {conv }}$ near the end of the simulation, and that lasts for duration $\Delta t$. For many models, there is actually no need to time-average because the time variability in $\Sigma_{\text {gap }}$ is fractionally small (less than a few percent). However, some models exhibit greater variability, particularly for $q$ approaching $10^{-2}$ or $h / r=0.1$. The fluctuations appear periodic, with periods ranging from $0.5-1 P_{\mathrm{p}}$ and amplitudes up to order unity. For these more strongly time-variable cases, we also record in Table 1 the maximum and minimum values of $\Sigma_{\text {gap }}$ that occur during the averaging interval.

\section{RESULTS}

In $\$ 3.1$ we obtain empirical scalings for gap depths at $10^{-4} \leq q \leq 5 \times 10^{-3}$; in $\$ 3.2$ we repeat for $5 \times 10^{-3} \leq q \leq$ $10^{-2}$ and discuss qualitatively the new dynamical phenomena that appear at these highest companion masses; and in $\$ 3.3$ we highlight some of the differences between PEnGUIn and ZEUS90.

\subsection{Gap Depth Scalings for $10^{-4} \leq q \leq 5 \times 10^{-3}$}

Gap depths $\Sigma_{\text {gap }}\left(\Sigma_{0}\right)$ are recorded in Table 1 and plotted against each of the parameters $q, \alpha$, and $h / r$ in Figures 6 , 7) and 8, respectively. Overall the agreement between the two codes, which utilize completely different algorithms, is remarkably good.

Figure 6 attests that for $q \lesssim 5 \times 10^{-3}$, gap depths scale roughly as $q^{-2}$ - as our analytic scaling (3) predicts. For $q \gtrsim 5 \times 10^{-3}$, the curves flatten somewhat (more on the behavior at large $q$ in $\$ 3.2$ ). In Figure 7, $\Sigma_{\text {gap }}$ appears to scale with $\alpha$ to a power between 1 and 1.5. For comparison, our analytic scaling (3) predicts $\Sigma_{\text {gap }} \propto \alpha^{1}$. The empirical dependence on $h / r$ is similarly steeper than the analytic dependence: equation (3) predicts that $\Sigma_{\text {gap }} \propto(h / r)^{5}$ whereas Figure 8 shows that the power-law indices vary between 5 and 7 .

We obtain a "best-fit" power-law relation by minimizing the function $y=\sum\left[\ln D q^{A} \alpha^{B}(h / r)^{C}-\ln \Sigma_{\text {gap }}\right]^{2} / N$ over the parameters $(A, B, C, D)$. The sum is performed over $N$ data points, excluding the discrepant runs at $q=0.01$ and runs for which $\Sigma_{\text {gap }} / \Sigma_{0}>0.2$ (i.e., runs for which gaps hardly open). With these exclusions, there are $N=13$ data points from PEnGUIn, best fitted by

$$
\begin{aligned}
10^{-4} \leq q & \leq 5 \times 10^{-3}: \\
\Sigma_{\text {gap }} / \Sigma_{0} & =0.14\left(\frac{q}{10^{-3}}\right)^{-2.16}\left(\frac{\alpha}{10^{-2}}\right)^{1.41}\left(\frac{h / r}{0.05}\right)^{6.61} .
\end{aligned}
$$

The $N=13$ points from ZEUS90 are best described by a very similar formula:

$$
\begin{aligned}
10^{-4} \leq q & \leq 5 \times 10^{-3}: \\
\Sigma_{\text {gap }} / \Sigma_{0} & =0.15\left(\frac{q}{10^{-3}}\right)^{-2.12}\left(\frac{\alpha}{10^{-2}}\right)^{1.42}\left(\frac{h / r}{0.05}\right)^{6.45} .
\end{aligned}
$$

Both of these relations fit their respective data to typically better than 20\%; the largest deviation in the PEnGUIn fit is $40 \%$, corresponding to $(q, \alpha, h / r)=\left(2 \times 10^{-3}, 10^{-3}, 0.05\right)$, and for ZEUS90 the largest deviation is $50 \%$, corresponding to $(q, \alpha, h / r)=\left(10^{-3}, 10^{-3}, 0.05\right)$.

Because our two codes agree so well, and because the fits are good, we are confident the deviations between our empirical scaling (say equation 12 from PEnGUIn) and our analytic scaling (3) are real and reflect physical effects not captured by our analytic scaling. And because our analytic scaling (3) matches exactly the scalings found numerically by Duffell and MacFadyen (2013) at low $q \lesssim 10^{-4}$ — whereas our empirical relation (12) applies to high $q \gtrsim 10^{-4}$ - these physical effects manifest for giant (Jupiter-like) planets, not lower-mass (Neptune-like) planets 7 We have not elucidated what this physics is, although there might be some clues from the gap behavior at the very highest values of $q$ we tested, as discussed in $\$ 3.2$.

At the same time, we emphasize that the deviations between (3) and (12), though (probably) real, are not large. If we insist on fitting the PEnGUIn data using (3) - i.e., if we fix $(A, B, C)=(-2,1,5)$ and allow only the coefficient $D$ to float - then the data deviate from (3) by typically a factor of 2 , and at most a factor of 3 . Thus the physical effects not captured by our analytic scaling, whatever they are, do not lead to order-of-magnitude changes in gap depth, at least over the range of parameters tested.

\subsection{Behavior of Gaps at High $q \gtrsim 5 \times 10^{-3}$}

The dependence of $\Sigma_{\text {gap }}$ on $q$ flattens at the highest values of $q$ considered (Figure 6). Fitting the $N=8$ points from PEnGUIn for which $q \geq 5 \times 10^{-3}$ and $\Sigma_{\text {gap }} / \Sigma_{0}<0.2$ yields:

$$
\begin{aligned}
& 10^{-2} \geq q \geq 5 \times 10^{-3}: \\
& \Sigma_{\text {gap }} / \Sigma_{0}=4.7 \times 10^{-3}\left(\frac{q}{5 \times 10^{-3}}\right)^{-1.00}\left(\frac{\alpha}{10^{-2}}\right)^{1.26}\left(\frac{h / r}{0.05}\right)^{6.12} .
\end{aligned}
$$

Similarly for the $N=8$ points from ZEUS90 we obtain:

$$
\begin{aligned}
& 10^{-2} \geq q \geq 5 \times 10^{-3}: \\
& \Sigma_{\text {gap }} / \Sigma_{0}=5.6 \times 10^{-3}\left(\frac{q}{5 \times 10^{-3}}\right)^{-1.02}\left(\frac{\alpha}{10^{-2}}\right)^{1.34}\left(\frac{h / r}{0.05}\right)^{6.12} .
\end{aligned}
$$

Although at first glance one might attribute the flattening of the trend of $\Sigma_{\text {gap }}$ with $q$ to the onset of strong shocks, we do not believe this is the correct interpretation. In the strongshock regime, where disturbances excited by the planet are

\footnotetext{
${ }^{7}$ Another difference between our simulations and theirs is that we mandate a steady $\dot{M} \neq 0$ across our entire domain, whereas they adopt (as appears customary for work in this field) wave-killing zones that effectively result in nearly zero-inflow boundary conditions. We have verified, however, that this difference does not matter for $\Sigma_{\text {gap }}$; we implemented zero-inflow boundaries in a few runs with PEnGUIn and found results for $\Sigma_{\text {gap }}$ that matched those with our standard accreting boundaries to better than $1 \%$.
} 

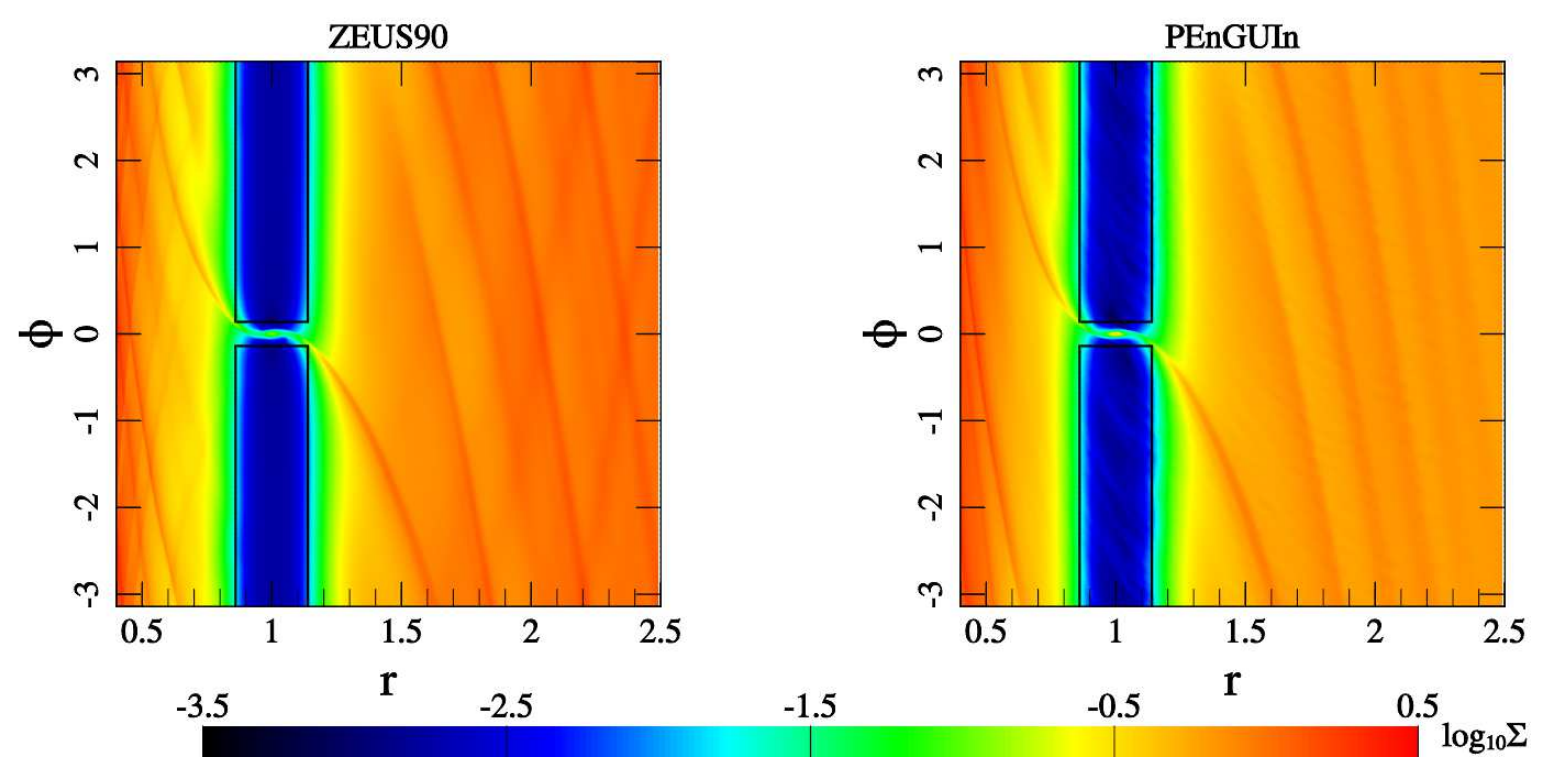

FIG. 3.- Snapshots of simulations with $(q, \alpha, h / r)=\left(10^{-3}, 10^{-3}, 0.05\right)$. PEnGUIn's snapshot is taken at $t=2 \times 10^{4} P_{\mathrm{p}}$ while ZEUS90's is taken at $t=1 \times 10^{4} P_{\mathrm{p}}$. Overall the two codes agree well on the shape and depth of the gap. ZEUS90 has more trouble converging to the desired outer boundary condition; $\Sigma$ at $r=2.5$ deviates from that imposed by equation (9) by up to $\sim 50 \%$. Note that PEnGUIn does not have the problem in the outer disk that ZEUS90 does, and moreover succeeds in resolving fine streamers ("filaments") within the gap. The black rectangles indicate the area over which $\Sigma_{\text {gap }}$ is averaged.
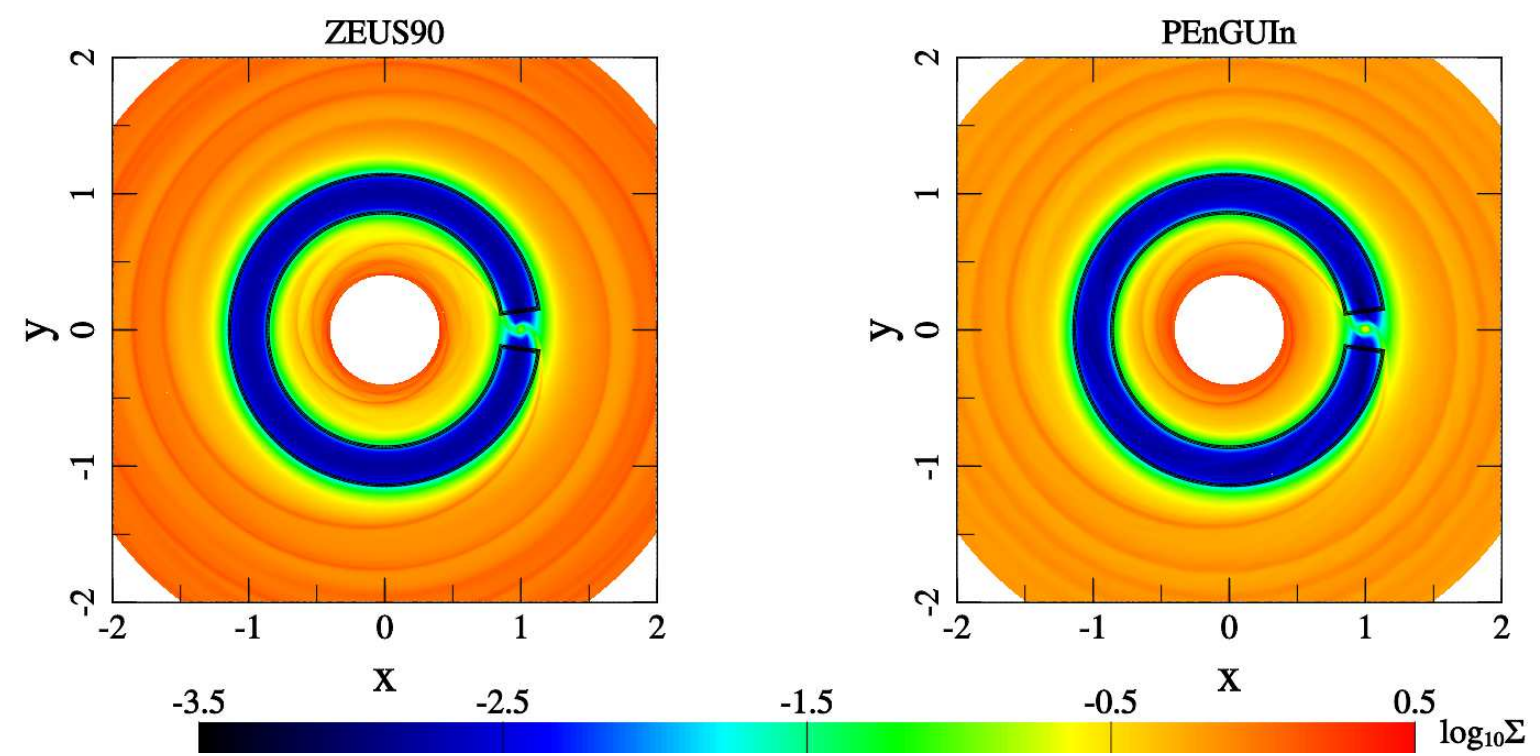

Fig. 4.- Cartesian version of Figure 3

non-linear at launch, the torque exerted on the disk by the planet scales as $q^{1}(h / r)^{0}$ (e.g., Hopkins and Ouataert 2011, their section 2.3) 8 Then the same arguments in $\$ 1.1$ yield $\Sigma_{\text {gap }} \propto q^{-1}(h / r)^{2}$. This analytic relation reproduces the scaling index for $q$ given by our empirical relations (14) and (15), but fails to reproduce the empirical scaling index for $h / r$. Furthermore, the flattening begins at an apparently "universal" $q$ -

\footnotetext{
8 This scaling can be seen by replacing $h$ with $R_{\mathrm{H}}$ in equation (1); the torque cut-off distance generally equals $\max \left(R_{\mathrm{H}}, h\right)$, which in the strongshock limit equals $R_{\mathrm{H}}$.
}

value of $\sim 5 \times 10^{-3}$ that is independent of $h / r$, whereas in the strong-shock interpretation, the critical $q$-value should scale as $(h / r)^{3}$ (i.e., the expected critical $q$ is given by the so-called thermal mass).

We do not have an explanation for the flatter slope of -1 at high $q$. We speculate that it might be caused by the most massive companions causing material at the gap edge to "leak" into the gap. The most massive planets disturb the gap edge so strongly that local instabilities send streamers of gas into the gap. These streamers, which de Val-Borro et al. (2006) called "filaments", are prominent in the high- $q$ snapshots in 
TABLE 1

Simulated Gap Depths

\begin{tabular}{|c|c|c|c|c|c|c|c|c|c|c|}
\hline \multirow[b]{2}{*}{$q$} & \multirow[b]{2}{*}{$\alpha$} & \multirow[b]{2}{*}{$h / r$} & PEnGUIn & ZEUS90 & \multicolumn{2}{|c|}{ PEnGUIn } & \multicolumn{2}{|c|}{ ZEUS90 } & \multirow{2}{*}{$\begin{array}{r}\text { PEnGUIn } \\
\text { Comn }\end{array}$} & \multirow{2}{*}{$\begin{array}{l}\text { ZEUS90 } \\
\text { lents }\end{array}$} \\
\hline & & & \multicolumn{2}{|c|}{$\Sigma_{\text {gap }}{ }^{\text {a }}\left(\Sigma_{0}\right)$} & $t_{\text {conv }}{ }^{\mathrm{b}}\left(P_{\mathrm{p}}\right)$ & $\Delta t^{\mathrm{b}}\left(P_{\mathrm{p}}\right)$ & $t_{\text {conv }}\left(P_{\mathrm{p}}\right)$ & $\Delta t\left(P_{\mathrm{p}}\right)$ & & \\
\hline $1 \times 10^{-4}$ & $10^{-3}$ & 0.05 & $4.6 \times 10^{-1}$ & $4.9 \times 10^{-1}$ & 20000 & 10 & 10000 & 10 & & d \\
\hline $2 \times 10^{-4}$ & $10^{-3}$ & 0.05 & $1.9 \times 10^{-1}$ & $2.0 \times 10^{-1}$ & 20000 & 10 & 10000 & 10 & & $\mathrm{~d}$ \\
\hline $5 \times 10^{-4}$ & $10^{-3}$ & 0.05 & $3.0 \times 10^{-2}$ & $3.1 \times 10^{-2}$ & 20000 & 10 & 10000 & 10 & & d \\
\hline $1 \times 10^{-3}$ & $10^{-3}$ & 0.05 & $4.0 \times 10^{-3}$ & $3.9 \times 10^{-3}$ & 20000 & 10 & 10000 & 10 & & \\
\hline $2 \times 10^{-3}$ & $10^{-3}$ & 0.05 & $8.5_{-0.3}^{+0.4} \times 10^{-4}$ & $1.2_{-0.2}^{+0.1} \times 10^{-3}$ & 6000 & 10 & 6000 & 10 & & $\mathrm{e}$ \\
\hline $5 \times 10^{-3}$ & $10^{-3}$ & 0.05 & $2.1_{-0.2}^{+0.2} \times 10^{-4}$ & $2.6_{-0.8}^{+0.5} \times 10^{-4}$ & 6000 & 10 & 6000 & 10 & $\mathrm{f}$ & $\mathrm{e}$ \\
\hline $1 \times 10^{-2}$ & $10^{-3}$ & 0.05 & $1.7_{-0.5}^{+0.4} \times 10^{-4}$ & $1.4_{-0.5}^{+0.8} \times 10^{-4}$ & 6000 & 10 & 6000 & 10 & $\mathrm{f}$ & \\
\hline $1 \times 10^{-3}$ & $10^{-2}$ & 0.05 & $1.4 \times 10^{-1}$ & $1.5 \times 10^{-1}$ & 2000 & 10 & 2000 & 10 & & \\
\hline $2 \times 10^{-3}$ & $10^{-2}$ & 0.05 & $2.7 \times 10^{-2}$ & $2.8 \times 10^{-2}$ & 2000 & 10 & 2000 & 10 & & \\
\hline $5 \times 10^{-3}$ & $10^{-2}$ & 0.05 & $5.5_{-10}^{+0.9} \times 10^{-3}$ & $4.6_{-18}^{+2.6} \times 10^{-3}$ & 2000 & 10 & 2000 & 10 & & \\
\hline $1 \times 10^{-2}$ & $10^{-2}$ & 0.05 & $2.0_{-0.8}^{+0.6} \times 10^{-3}$ & $2.7_{-0.4}^{+0.6} \times 10^{-3}$ & 2000 & 10 & 2000 & 10 & $\mathrm{e}$ & \\
\hline $1 \times 10^{-3}$ & $10^{-1}$ & 0.05 & $6.1 \times 10^{-1}$ & $7.2 \times 10^{-1}$ & 300 & 10 & 300 & 10 & & \\
\hline $2 \times 10^{-3}$ & $10^{-1}$ & 0.05 & $3.5 \times 10^{-1}$ & $4.9 \times 10^{-1}$ & 300 & 10 & 300 & 10 & & \\
\hline $5 \times 10^{-3}$ & $10^{-1}$ & 0.05 & $9.8 \times 10^{-2}$ & $1.7 \times 10^{-1}$ & 300 & 10 & 300 & 10 & & \\
\hline $1 \times 10^{-2}$ & $10^{-1}$ & 0.05 & $3.8 \times 10^{-2}$ & $4.9_{-0.2}^{+0.2} \times 10^{-2}$ & 300 & 10 & 300 & 10 & & \\
\hline $1 \times 10^{-3}$ & $10^{-3}$ & 0.1 & $2.2 \times 10^{-1}$ & $2.4_{-0.1}^{+0.1} \times 10^{-1}$ & 7000 & 10 & 7000 & 10 & & \\
\hline $5 \times 10^{-3}$ & $10^{-3}$ & 0.1 & $1.5_{-0.1}^{+0.5} \times 10^{-2}$ & $1.6_{-0.1}^{+0.1} \times 10^{-2}$ & 6000 & 1000 & 7000 & 10 & $\mathrm{c}$ & \\
\hline $1 \times 10^{-2}$ & $10^{-3}$ & 0.1 & $8.4_{-0.6}^{+0.5} \times 10^{-3}$ & $1.0_{-0.1}^{+0.1} \times 10^{-2}$ & 6900 & 100 & 7000 & 10 & c & \\
\hline $5 \times 10^{-4}$ & $10^{-3}$ & 0.04 & $6.0 \times 10^{-3}$ & $6.5 \times 10^{-3}$ & 17000 & 10 & 10000 & 10 & & $\mathrm{~d}$ \\
\hline $2 \times 10^{-3}$ & $10^{-3}$ & 0.04 & $2.7_{-0.2}^{+0.2} \times 10^{-4}$ & $2.9_{-0.6}^{+0.6} \times 10^{-4}$ & 10000 & 10 & 5000 & 10 & & $\mathrm{e}$ \\
\hline $2 \times 10^{-3}$ & $10^{-2}$ & 0.04 & $7.9_{-0.9}^{+1.1} \times 10^{-3}$ & $7.0_{-0.5}^{+0.4} \times 10^{-3}$ & 4000 & 10 & 4000 & 10 & & \\
\hline
\end{tabular}

${ }^{a}$ Averaged over time and over a partial annulus centered on the planet, as defined in $\$ 2.3 .4$ and delineated in Figures 3 and 4 For visibly eccentric outer disks (see Comments column), the outer edge of the measurement annulus is made eccentric to conform to the gap shape (e.g., Figures 10 and 11. Maximum and minimum values of $\Sigma_{\text {gap }}$ are given for runs for which these values deviate from the time-averaged value by more than a percent. All surface densities are in units where $\Sigma(r=1)=1$ in a steadily accreting, planet-less disk.

$\mathrm{b}$ The time $t_{\text {conv }}$ is taken near the end of a simulation, when $\Sigma_{\text {gap }}$ appears to have nearly converged to its steady-state value (see Figure 5 . Formally $t_{\text {conv }}$ marks the beginning of the time interval, of duration $\Delta t$, over which $\Sigma_{\text {gap }}$ is averaged. All times listed are in units of the planetary orbital period, $P_{\mathrm{p}}$.

${ }^{\mathrm{c}}$ Highly unsteady outer gap edge (e.g., Figure 9 and long-term time variability. A longer $\Delta t$ is chosen to capture the variability.

${ }^{\mathrm{d}}$ Indirect potential included $(\$ 2.2$.

e Eccentric outer disk (e.g., Figures 10 and 11 . Measured eccentricities are $\sim 0.10-0.15$ and apsidal precession periods are $\sim 300-600 P_{p}$.

${ }^{\mathrm{f}}$ An eccentric outer disk is observed at $t \sim 1000 P_{\mathrm{p}}$, but the eccentricity damps away by $t_{\text {conv }}$. The damping is probably artificial ( $\$ 3.2$.

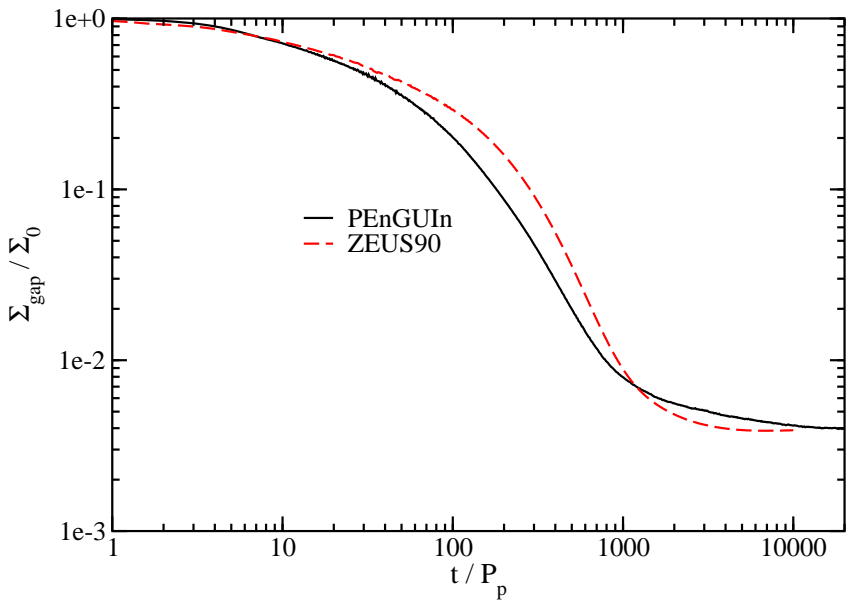

Fig. 5.- Convergence of $\Sigma_{\text {gap }}$ with time for $(q, \alpha, h / r)=\left(10^{-3}, 10^{-3}, 0.05\right)$. For these parameters, the viscous timescale is formally $r_{\mathrm{p}}^{2} / v \sim 6 \times 10^{4}$ planetary orbits.

Figure 9 (and can be seen even at $q=10^{-3}$ in the PEnGUIn snapshot in Figure 3). The filaments appear to originate from unsteady structures along gap edges; similar structures were seen by, e.g., Kley \& Dirksen (2006, their figures 1 and 7).

We also observe evidence for an eccentric outer disk at high $q$; see Figure 10 and the "Comments" column in Table1 The

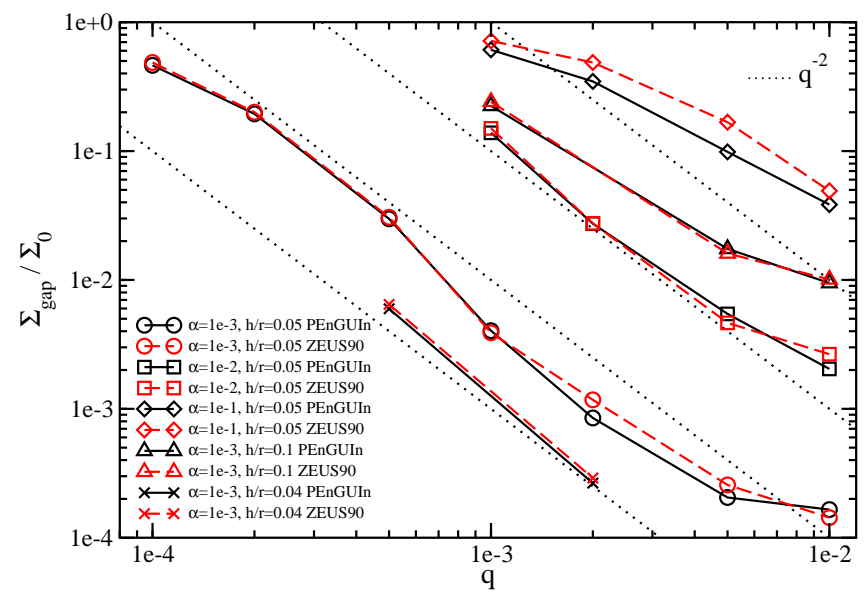

FIG. 6. $-\Sigma_{\text {gap }}$ vs. $q$. Black dotted lines indicate constant power-law slopes of -2 , and are shown for reference only. The power-law slopes approximately equal -2 for $q<5 \times 10^{-3}$, and flatten to -1 for higher $q$. For formal powerlaw fits, see the main text.

outward transport of angular momentum by waves launched at the 1:3 outer eccentric Lindblad resonance pumps the eccentricity of the outer disk (Lubow 1991; Papaloizou et al. 2001). The $q$-value for which disks become eccentric depends on $\alpha, h / r$, and disk mass (Lubow 1991; Papaloizou et al. 2001; Kley and Dirksen 2006; D'Angelo et al. 2006; Dunhill et al. 


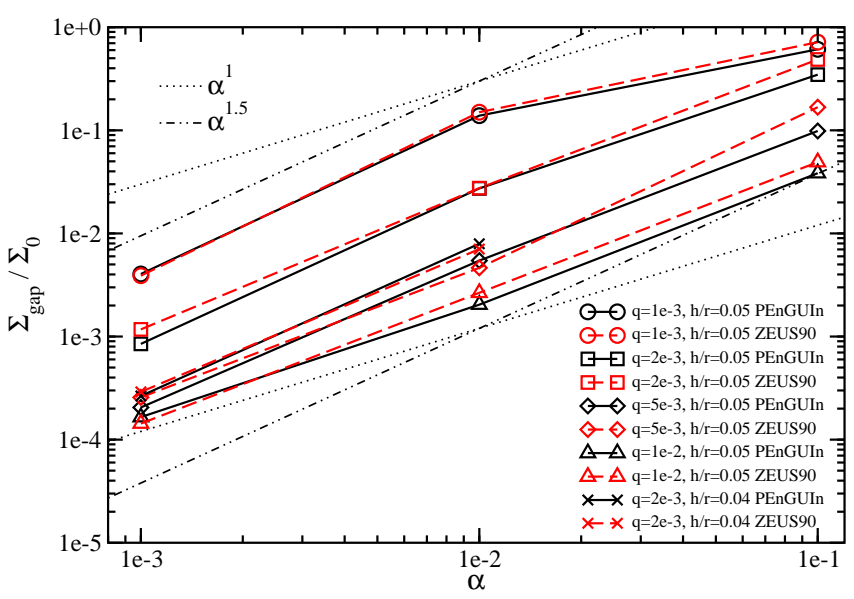

FIG. 7.- $\Sigma_{\text {gap }}$ vs. $\alpha$. Dotted and dot-dot-dashed lines indicate power-law slopes of 1 and 1.5 , bracketing the range exhibited by the data. For formal power-law fits, see main text.

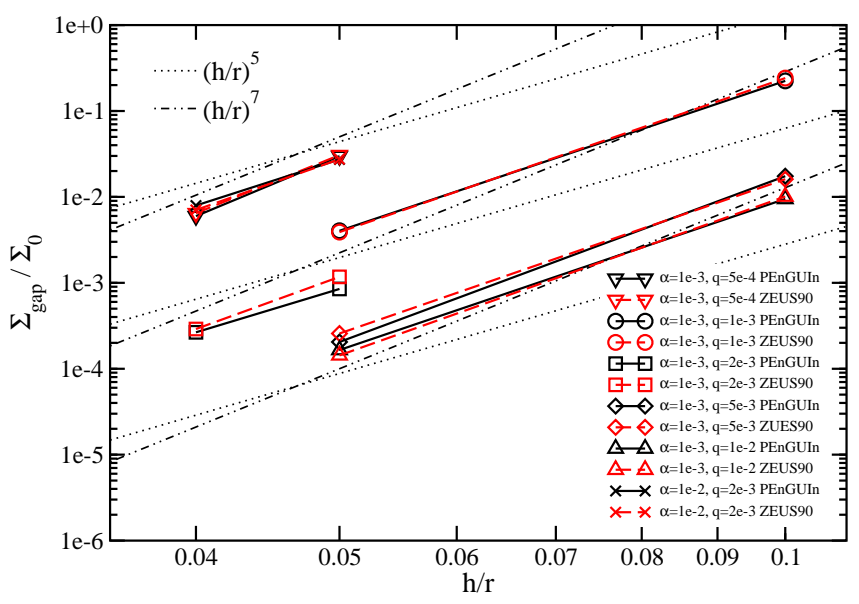

FIG. 8. $-\Sigma_{\text {gap }}$ vs. $h / r$. Dotted and dot-dot-dashed lines indicate power-law slopes of 5 and 7 , bracketing the range exhibited by the data. For formal power-law fits, see main text.

2013). For a planet held on a fixed circular orbit embedded in a non-gravitating disk for which $h / r=0.05$ and $\alpha \approx 0.005$, Kley and Dirksen (2006) found that $q \gtrsim 0.003$ led to eccentric disks; for $\alpha \approx 0.01$, the required $q \gtrsim 0.005$. Their findings are in line with ours.

In two runs with PEnGUIn, an eccentricity appears in the outer disk at early times but damps away by the time $\Sigma_{\text {gap }}$ converges (see Table 1). The eccentricity damping is probably an artifact of our outer circular boundary at $r_{\text {out }}=2.5 r_{\mathrm{p}}$, which according to Kley and Dirksen (2006) is too close to the planet to properly simulate eccentric disks. The danger posed by the outer boundary should lessen as the $\alpha$-viscosity increases and disturbances excited by the planet are more localized; this may be why circularization occurs only for our lowest $\alpha=10^{-3}$ runs at high $q$.

We note that the fine-structure filaments threading the gaps are seen in most of our $q \geq 0.001$ simulations, while only a few of these cases evince eccentric outer disks. Moreover, the filaments observed by de Val-Borro et al. (2006) do not appear associated with disk eccentricity. It is unclear to us whether the filaments and disk eccentricity are directly related.

\subsection{Code Comparison}

Generally PEnGUIn and ZEUS90 agree very well on $\Sigma_{\text {gap }}$ (e.g., Figures 6 8), typically differing by no more than a few tens of percent, and often much better. Because the codes rely on fundamentally different algorithms - one is a shockcapturing Lagrangian-remap code, while the other is an Eulerian code using the upwind method - their agreement lends confidence in the accuracy of our results. Some minor, systematic differences include: (i) $\Sigma_{\text {gap }}$ for ZEUS90 is larger than for PEnGUIn; (ii) PEnGUIn usually resolves a higher density peak near the planet; (iii) PEnGUIn typically requires a longer time to converge; and (iv) near the outer boundary where the resolution is coarser, ZEUS90 has difficulty relaxing to the steadily accreting solution described by equations (9)-(11), deviating from the correct $\Sigma$ by up to $50 \%$. All of these differences may be attributed to the fact that PEnGUIn uses PPM, which is a fourth-order method for uniform grids (third-order for non-uniform grids), whereas ZEUS90's algorithm is only second-order in space. Thus PEnGUIn tends to be more accurate and less numerically diffusive than ZEUS90, at the cost of taking a longer time to resolve sharp features.

A key innovation of PEnGUIn is its use of GPU technology to accelerate computations. PEnGUIn's speed on a single GTX-Titan graphics card can rival that of a traditional CPU cluster having $\sim 100$ cores. For this paper we ran PEnGUIn on a desktop computer housing 3 graphics cards. With specialized hardware we can connect up to 8 GPUs to a motherboard. PEnGUIn's scalability with the number of cards approaches linear as the resolution increases; our speed on 3 cards is 2.33 $\times$ that of a single card at our standard resolution; if the resolution is doubled (quadrupled), the speed enhancement factor increases to 2.64 (2.92) as PEnGUIn takes more full advantage of GPU's parallelism. Currently PEnGUIn can run on a single node only, and would need to be modified to run on multiple nodes. In a multi-node cluster of GPUs, the scaling of speed with the number of cards per node is unlikely to be linear because communication between nodes is significantly slower than between cards on a single node.

\section{CONCLUSIONS AND OUTLOOK}

We established two empirical formulas (12 and 14) for the surface density contrast, $\Sigma_{\text {gap }} / \Sigma_{0}$, inside and outside the gap carved by a non-accreting giant planet. The first is valid for planet-to-star mass ratios $10^{-4} \leq q \leq 5 \times 10^{-3}$, and the second is valid for $5 \times 10^{-3} \leq q \leq 10^{-2}$. Our formulae are derived from our new, fast, Lagrangian shock-capturing PPM code PEnGUIn, and are confirmed by ZEUS90. Combining our results with those from the literature, we find that $\Sigma_{\text {gap }}$ scales with $q$, viscosity parameter $\alpha$, and disk aspect ratio $h / r$ in the following ways:

- At Neptune-like (and perhaps lower) masses, Duffell and MacFadyen (2013) found that $\Sigma_{\text {gap }} \propto q^{-2} \alpha^{1}(h / r)^{5}$

- At Jupiter-like masses, we find that $\Sigma_{\text {gap }} \propto$ $q^{-2.2} \alpha^{1.4}(h / r)^{6.6}$ (our equation 12 );

- At masses near the brown dwarf threshold, we find that $\Sigma_{\text {gap }} \propto q^{-1} \alpha^{1.3}(h / r)^{6.1}$ (our equation 14).

${ }^{9}$ A caveat is that Duffell and MacFadyen (2013) did not explicitly test the dependence on $h / r$ that they proposed. We used PEnGUIn to try to reproduce their low-mass results (data not shown), but encountered the problem that $\Sigma_{\text {gap }}$ took too long to converge. What low-mass data we did collect at the end of 20000 orbital periods were consistent with the power-law indices proposed by Duffell and MacFadyen (2013). 

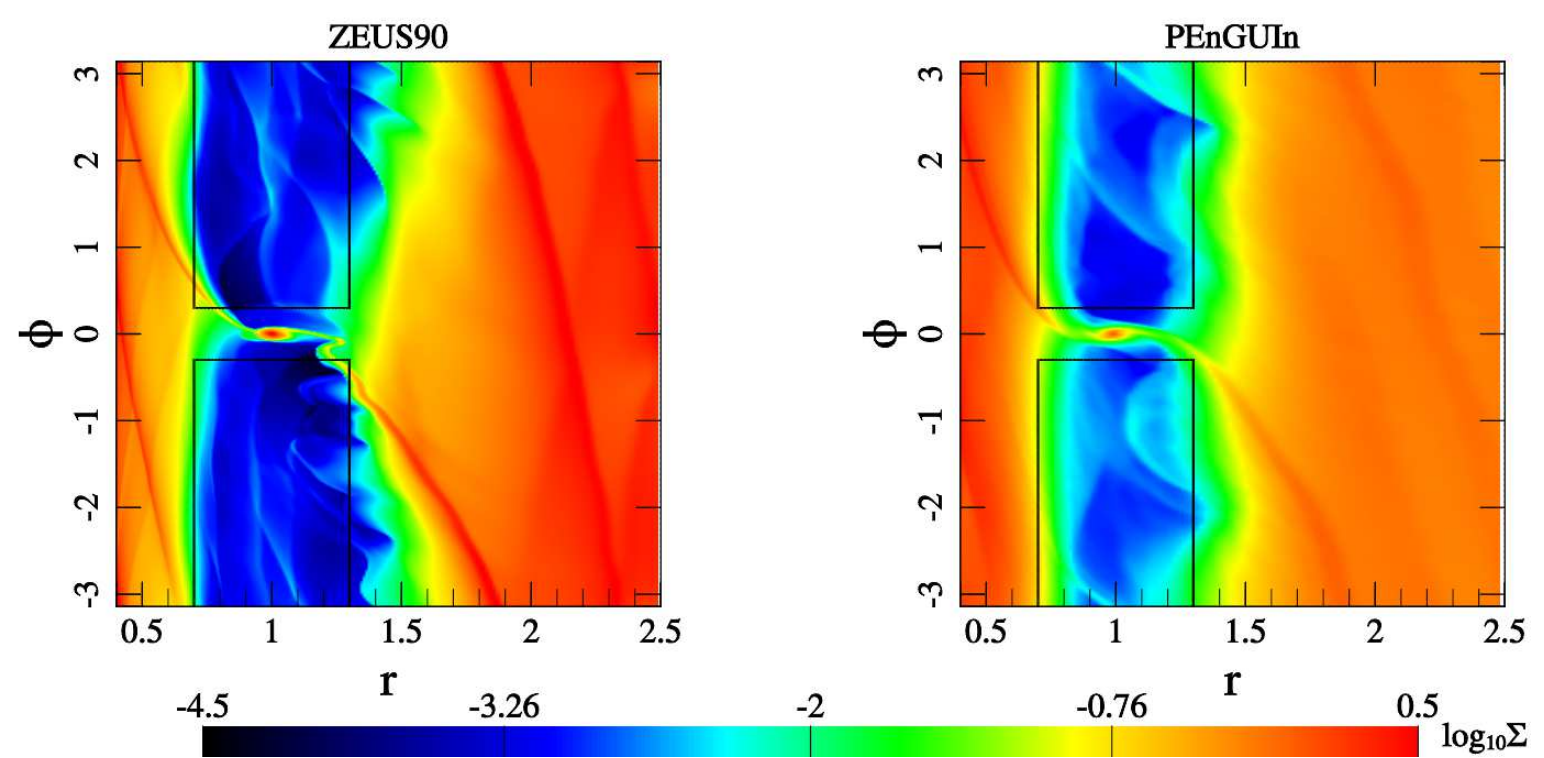

FIG. 9.- Two different examples at high $q$ of unsteady gap edges and streamers filling gaps. The ZEUS90 snapshot is for $(q, \alpha, h / r)=(0.01,0.01,0.05)$ and the PEnGUIn snapshot is for $(q, \alpha, h / r)=(0.01,0.001,0.1)$.
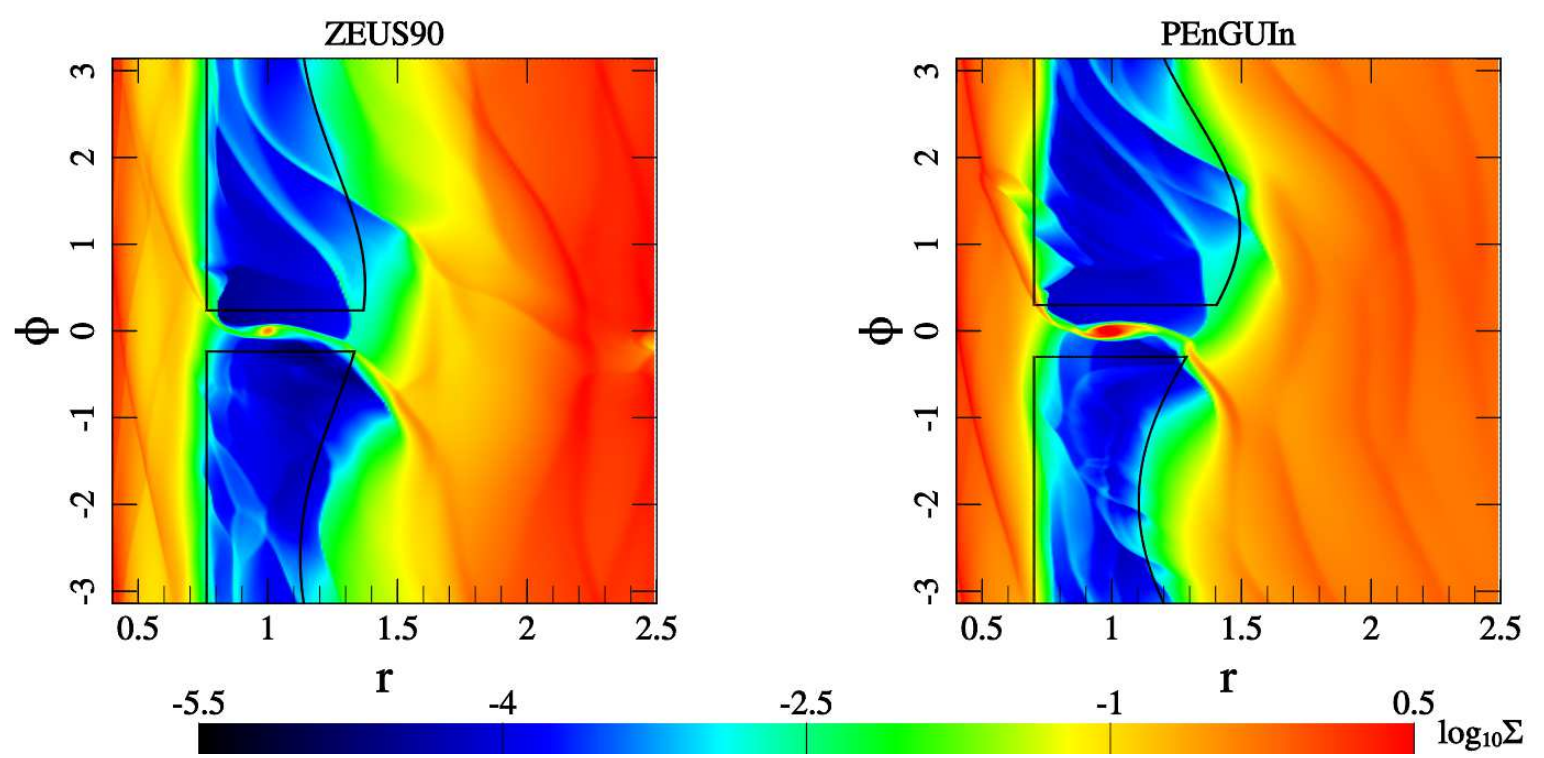

FIG. 10.- Snapshots of eccentric outer disks, one from ZEUS90 at $(q, \alpha, h / r)=(0.005,0.001,0.05)$, and another from PEnGUIn at $(q, \alpha, h / r)=(0.01,0.01,0.05)$. For the ZEUS90 run shown, the inner edge of the outer disk (exterior to the planet's orbit) has eccentricity 0.10 and precession period $630 P_{\mathrm{p}}$. For the PEnGUIn run, the eccentricity is 0.15 and the precession period is $380 P_{\mathrm{p}}$. Black curves enclose the area over which $\Sigma_{\text {gap }}$ is computed.

Our scaling indices for giant planets and quasi-brown dwarfs are supported by two independent codes using different algorithms, and so we are confident in their accuracy. Note that our simulations and those of Duffell and MacFadyen (2013) do share one common set of parameters: $(q, \alpha, h / r) \approx(5 \times$ $\left.10^{-4}, 10^{-3}, 0.05\right)$, for which we find $\Sigma_{\text {gap }} / \Sigma_{0}=0.03$ and they find $\Sigma_{\text {gap }} / \Sigma_{0}=0.04$ (their Figure 2) 10 We consider this good

10 Technically, our simulations have spatially constant $\alpha$ and $h / r$, whereas theirs has spatially constant $v=\alpha c_{\mathrm{s}} h$ and $h / r \propto r^{0.25}$. Also, we compute $\Sigma_{\text {gap }}$ as an area average, whereas they report the minimum surface density. These agreement.

The scaling differences between low mass and high mass, although pointing to real physical effects, do not lead to orderof-magnitude changes in gap depth, at least over the range of parameters surveyed. That is, using the Neptune-like scaling $\Sigma_{\text {gap }} \propto q^{-2} \alpha^{1}(h / r)^{5}$ for Jupiter-like planets leads to gap depths that differ (systematically) from those observed in our simulations by factors of only $2-3$.

differences are probably immaterial. 

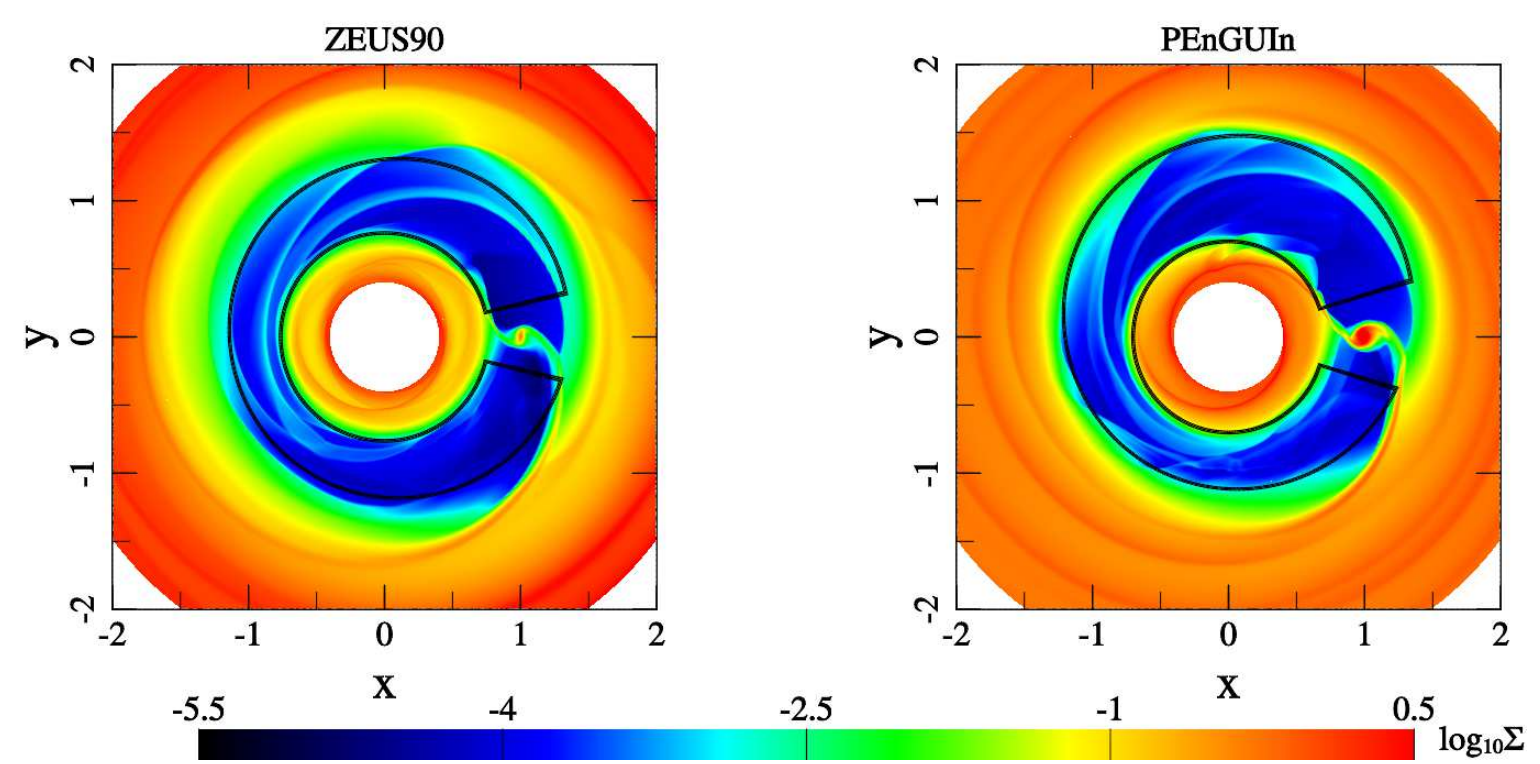

FIg. 11.- Cartesian view of the eccentric disks of Figure 10

\subsection{Connecting to Observations of Transition Disks}

Are the gaps empty enough to reproduce the low optical depths characterizing the cavities of transitional and pre-transitional disks? Surface density contrasts from models of disks like PDS 70 (Dong et al. 2012) and GM Aur (Calvet et al. 2005) are $10^{3}$ or more. We have found that certain sets of planet-disk parameters can achieve such contrasts. For example, $(q, \alpha, h / r)=\left(5 \times 10^{-3}, 10^{-3}, 0.05\right)$ produces contrasts of $\sim 5000$ (Table 11). Lower mass planets could also be made to work with lower $\alpha$-viscosities and/or cooler disks with lower $h / r$; as a further example, $(q, \alpha, h / r)=$ $\left(2 \times 10^{-3}, 10^{-3}, 0.04\right)$ generates a contrast of $\sim 3000$. The dependence on disk temperature is especially sensitive: $\Sigma_{\text {gap }} \propto$ $(h / r)^{6.6} \propto T^{3.3}$.

The surface density contrasts reported in this paper are all underestimates insofar as we have neglected accretion onto the planet; but arguably the disk accretion rate cannot be reduced by more than a factor of order unity, lest the planet starve the host star and violate observed stellar accretion rates (Zhu et al. 2011; see also Lubow and D'Angelo 2006). We would argue further that our gas surface density contrasts are also underestimates of dust surface density (i.e., optical depth) contrasts, to the extent that mechanisms like dust filtration at outer gap edges (e.g., Zhu et al. 2012) deplete dust relative to gas in gaps.

Given these findings, we feel that when it comes to transition disks, the problem is not so much gap depth, but gap width. A single planet embedded in an accreting disk generates a gap too narrow in radial width to explain the expansive cavities observed in transition disks. To connect to observations would seem to require that we expand our study to include multiple planets or brown dwarfs within a viscous, gravitating disk, as has been done by Zhu et al. (2011). These authors discounted $\alpha \lesssim 0.002$ - and were therefore compelled to invoke additional channels of opacity reduction (e.g., grain growth) to explain transition disks - because multiple planets were found to be dynamically unstable at low $\alpha /$ high $\Sigma$ (see their page 8 ). The incompatibility of multiple giant plan- ets with low $\alpha$ is a result that we would like to see confirmed independently and further explored.

\subsection{A Floor on $\Sigma_{\text {gap }}$}

All our empirical scalings for $\Sigma_{\text {gap }}$ suggest that arbitrarily low values of $\alpha$ generate arbitrarily clean gaps. We expect, however, the scalings to break down for small enough $\alpha$. Without an intrinsic disk viscosity, a planet may stir the gap edge in such a way as to trigger local instabilities and turbulent diffusion. For example, with $\alpha=0$, the outer gap edge might be so sharp as to be Rayleigh unstable. There should therefore be a minimum value or "floor" on $\Sigma_{\text {gap }}$ caused by a minimum planet-driven viscosity. Just such a floor has been reported by Duffell and MacFadyen (2013); see their Figure 7. Other simulations of planets in inviscid disks, concentrating on orbital migration and not gap depth, have been carried out by Li et al. (2009) and Yu et al. (2010).

Similar arguments suggest that there might also be a floor on $\Sigma_{\text {gap }}$ at high $q$. The streamers/filaments that invade the gap are densest for the highest $q$-values we tested.

\subsection{Analytic Derivation}

In \$1.1, we presented an analytic derivation of gap depth $\Sigma_{\text {gap }}$. We discovered that the power-law scalings in our analytic relation (3) match precisely those reported from numerical experiments for low-mass planets by Duffell and MacFadyen (2013). Our analytic relation can even reproduce approximately (deviating systematically by factors of a few) the empirical results we found for gaps carved by Jupiter-like planets.

The success of our breezy analytic derivation for $\Sigma_{\text {gap }}$ is surprising. Our derivation is "zero-dimensional" ("OD") because it considers only the total rates of angular momentum transport - a.k.a. the total "angular momentum luminosity" or "angular momentum current" - integrated over all azimuth and radius; it ignores the complicated details of how the torques are actually applied differentially in space. We have already noted in $\$ 1.1$ how it is not completely obvious that $\Sigma_{0}$ characterizes the viscous torques in gap edges. Furthermore, our 
OD treatment considers only the wave and viscous contributions to the total angular momentum current, and neglects the contribution from advection (i.e., the transport of angular momentum associated with a non-zero radial velocity $v_{r}$ ) and the contribution from azimuthal pressure variations (Crida et al. 2006).

We can address some of these problems and make the leap from $0 \mathrm{D}$ to $1 \mathrm{D}$ by considering the azimuthally averaged, radially dependent torque balance equation for a steady-state accretion disk perturbed by a planet (see, e.g., equation 3 of Lubow and D'Angelo 2006):

$$
\frac{d\left(3 v \Sigma \Omega r^{2}\right)}{d r}=-\Sigma \Omega r^{2} v_{r}+2 \Sigma r \Lambda(r)
$$

where the Lindblad torque per unit mass is

$$
\Lambda(r)=\operatorname{sgn}\left(r-r_{\mathrm{p}}\right) \frac{f G M_{*} q^{2}}{2 r}\left(\frac{r}{r-r_{\mathrm{p}}}\right)^{4}
$$

and $f$ is an order-unity constant. The left-hand side of 16 accounts for the viscous torque, while the first term on the right-hand side accounts for angular momentum transport by advection. We define $x \equiv r-r_{\mathrm{p}}$ and $\dot{M} \equiv 2 \pi \Sigma v_{r} r=$ constant $<0$, and approximate $\Omega=\Omega_{\mathrm{p}}\left(r / r_{\mathrm{p}}\right)^{-3 / 2}$ so that $v \Omega r=$ $v_{\mathrm{p}} \Omega_{\mathrm{p}} r_{\mathrm{p}}=$ constant (variables subscripted by $p$ take their values at the planet's orbital radius). For the outer disk, equation (16) simplifies to

$$
\alpha\left(\frac{h}{r}\right)^{2} \frac{d(r \Sigma)}{d r}=\frac{|\dot{M}| \Omega_{\mathrm{p}} r_{\mathrm{p}}}{6 \pi G M_{*}}\left(\frac{r}{r_{\mathrm{p}}}\right)^{-1 / 2}+\frac{f}{3} q^{2}\left(\frac{r}{x}\right)^{4} \Sigma .
$$

From this equation it becomes apparent how the outer accretion disk responds when repelled outward by a planet: for $q>0$, the surface density gradient $d \Sigma / d r$ steepens, just enough that the viscous torque can exceed the Lindblad torque and maintain a steady flow of mass inward (i.e., carry the $\dot{M}$ imposed at infinity across the planet's orbit).

Setting $\dot{M}=0$, and working in the WKB limit where $d / d r \gg 1 / r$, gives the standard zero-inflow solution: an exponential profile for $\Sigma(r)$, commonly used in the literature (e.g., Lubow et al. 1999; de Val-Borro et al. 2007; Mulders et al. 2013). Keeping $\dot{M} \neq 0$ alters $\Sigma(r)$ : it still resembles an exponential but is shifted outward (for fixed $f$ ), as Figure 12 demonstrates. We find for the parameters chosen that equation (18) describes well the gap profile from our 2D simulations, down to a distance of $\sim 3-4$ Hill radii away from the planet. But inside this cut-off distance, the 1D solution fails critically — it falls much too steeply to recover the actual flat-bottomed gap.

The problem of determining the gap depth analytically in 1D appears tantamount to the problem of understanding what happens inside this cut-off distance. Lindblad torques shut off here; the tidal gravitational field of the planet is especially strong; and circulating streamlines give way to horseshoe or- bits. One-dimensional analytic treatments may be inadequate to the task of modeling how gas navigates from the outer disk to the inner disk through a series of "horseshoe turns" (Lubow et al. 1999; Kley and Nelson 2012). As far as analytic treatments go, it may be that to do better than $0 \mathrm{D}$ requires at least $2 \mathrm{D}$.

Early explorations of this problem benefited from Steve Lubow, Daniel Perez-Becker, and the participants of the 2011

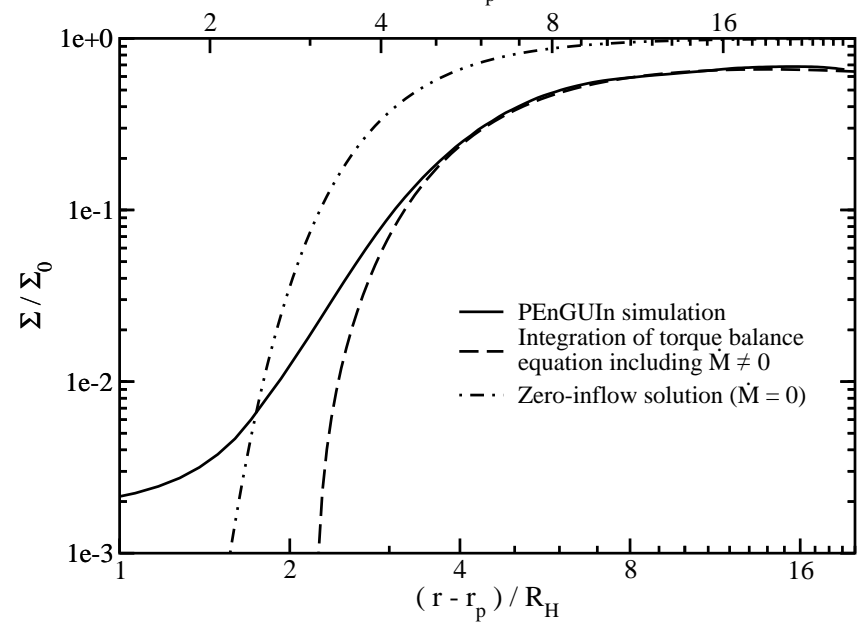

FIG. 12.- Reproducing the simulated gap profile with a 1D analysis. The solid curve is the azimuthally averaged surface density profile outside the planet's orbit for $(q, \alpha, h / r)=(0.001,0.001,0.05)$, as calculated from 2D simulations using PEnGUIn. Directly integrating the 1D equation (18) reproduces well the onset of the gap, if we set $f=0.2$ (dashed curve). However, the bottom of the gap is not captured at all. Setting $\dot{M}=0$, as is commonly done in the literature, yields a profile that is similar in shape to the actual profile, but shifted in radius (for the same value of $f=0.2$; dot-dot-dashed curve).

International Summer Institute for Modeling in Astrophysics (ISIMA) program organized by Pascale Garaud at the Kavli Institute for Astronomy and Astrophysics in Beijing University. We thank Meredith Hughes and Re'em Sari for encouraging discussions, and Paul Duffell, Andrew MacFadyen, Roman Rafikov, Miguel de Val-Borro, Zhaohuan Zhu, and especially an anonymous referee for thoughtful comments that led to substantive improvements. JF would like to especially thank Pawel Artymowicz for invaluable advice. Resources supporting this work were provided by the NASA High-End Computing (HEC) Program through the NASA Advanced Supercomputing (NAS) Division at Ames Research Center. Part of the simulations were performed with the Berkeley cluster Henyey, which was made possible by a National Science Foundation Major Research Instrumentation (NSF MRI) grant. Financial support was provided by a NASA Origins grant.

\section{REFERENCES}

P. Goldreich and S. Tremaine, ApJ 241, 425 (1980).

A. L. Kraus and M. J. Ireland, ApJ 745, 5 (2012).

J. H. Debes, H. Jang-Condell, A. J. Weinberger, A. Roberge, and G. Schneider, ApJ 771, 45 (2013).

S. P. Quanz, H. Avenhaus, E. Buenzli, A. Garufi, H. M. Schmid, and S. Wolf, ApJ 766, L2 (2013).

Z. Zhu, R. P. Nelson, L. Hartmann, C. Espaillat, and N. Calvet, ApJ 729, 47 (2011).

S. E. Dodson-Robinson and C. Salyk, ApJ 738, 131 (2011).
Z. Zhu, R. P. Nelson, R. Dong, C. Espaillat, and L. Hartmann, ApJ 755, 6 (2012).

R. Dong, J. Hashimoto, R. Rafikov, Z. Zhu, B. Whitney, T. Kudo, T. Muto, T. Brandt, M. K. McClure, J. Wisniewski, et al., ApJ 760, 111 (2012). W. Kley and R. P. Nelson, ARA\&A 50, 211 (2012).

W. R. Ward, ApJ 482, L211 (1997).

A. Crida, A. Morbidelli, and F. Masset, Icarus 181, 587 (2006).

S. H. Lubow and G. D'Angelo, ApJ 641, 526 (2006).

J. M. Stone and M. L. Norman, ApJS 80, 753 (1992). 
J. Frank, A. King, and D. Raine, Accretion Power in Astrophysics (Cambridge Astrophysics) (Cambridge University Press, 2002), ISBN 0521620538.

P. C. Duffell and A. I. MacFadyen, ApJ 769, 41 (2013).

N. I. Shakura and R. A. Sunyaev, A\&A 24, 337 (1973).

P. Colella and P. R. Woodward, Journal of Computational Physics 54, 174 (1984).

J. M. Blondin and E. A. Lufkin, ApJS 88, 589 (1993).

D. S. Balsara, ApJ 420, 197 (1994).

W. Kley, A\&A 338, L37 (1998).

J. F. Hawley and J. M. Stone, Computer Physics Communications 89, 127 (1995).

J.-M. Shi, J. H. Krolik, S. H. Lubow, and J. F. Hawley, The Astrophysical Journal 749, 118 (2012).

K. A. Sorathia, J. H. Krolik, and J. F. Hawley, The Astrophysical Journal 768, 133 (2013).

S. H. Lubow, M. Seibert, and P. Artymowicz, ApJ 526, 1001 (1999).
M. de Val-Borro, R. G. Edgar, P. Artymowicz, P. Ciecielag, P. Cresswell, G. D'Angelo, E. J. Delgado-Donate, G. Dirksen, S. Fromang,

A. Gawryszczak, et al., MNRAS 370, 529 (2006).

J. Goodman and R. R. Rafikov, ApJ 552, 793 (2001)

T. W. A. Müller, W. Kley, and F. Meru, A\&A 541, A123 (2012).

P. F. Hopkins and E. Quataert, MNRAS 415, 1027 (2011).

W. Kley and G. Dirksen, A\&A 447, 369 (2006).

S. H. Lubow, ApJ 381, 259 (1991).

J. C. B. Papaloizou, R. P. Nelson, and F. Masset, A\&A 366, 263 (2001).

G. D’Angelo, S. H. Lubow, and M. R. Bate, ApJ 652, 1698 (2006).

A. C. Dunhill, R. D. Alexander, and P. J. Armitage, MNRAS 428, 3072 (2013).

N. Calvet, P. D’Alessio, D. M. Watson, R. Franco-Hernández, E. Furlan, J. Green, P. M. Sutter, W. J. Forrest, L. Hartmann, K. I. Uchida, et al., ApJ 630, L185 (2005).

H. Li, S. H. Lubow, S. Li, and D. N. C. Lin, ApJ 690, L52 (2009).

C. Yu, H. Li, S. Li, S. H. Lubow, and D. N. C. Lin, ApJ 712, 198 (2010).

M. de Val-Borro, P. Artymowicz, G. D’Angelo, and A. Peplinski, A\&A 471, 1043 (2007).

G. D. Mulders, S.-J. Paardekooper, O. Panić, C. Dominik, R. van Boekel, and T. Ratzka, A\&A 557, A68 (2013). 